\title{
Association of ectodermal dysplasia, ectrodactyly, and macular dystrophy: the EEM syndrome
}

\author{
SHOZO OHDO, KIYOTAKE HIRAYAMA, AND TAMOTSU TERAWAKI
}

From the Department of Pediatrics, Miyazaki Medical College; the Department of Pediatrics, Faculty of Medicine, Ryukyu University; and the Department of Pediatrics, Faculty of Medicine, Kagoshima University, Japan.

SUMMARY We report five patients with ectodermal dysplasia, ectrodactyly associated with syndactyly or cleft hand or both, and, in addition, macular dystrophy which was presumed to be progressive, in $N$ an isolated population on a remote island in Japan. The heredity of this syndrome was thought to be응 autosomal recessive. Three cases have been reported so far with a combination of the same abnor- $\rightarrow$ malities. The parents in these cases were consanguineous. ${ }^{12}$

Among the syndromes of ectodermal dysplasia associated with malformations of the extremities, there are Roberts's syndrome, ${ }^{2}$ EEC syndrome, ${ }^{4}$ the syndrome reported by Robinson et al, ${ }^{5}$ the syndrome reported by Freire-Maia, ${ }^{6}$ and the syndrome reported by Bowen and Armstrong. ${ }^{7}$ However, these syndromes clearly differ from the syndrome described in this paper, because they lack the characteristic findings in the ocular fundus.

\section{Case reports}

CASE 1

The proband $(\mathrm{V} \cdot 1$, fig 1$)$ was a $16 \frac{3}{4}$-year-old girl when Received for publication 12 June 1982. we first examined her. Her parents were first cousins $<$ and had no signs of ectrodactyly or ectodermal $\overrightarrow{0}$ dysplasia. Her two sibs were healthy.

On physical examination, her hair was very sparse, thin, and short and the eyebrows and eyelashes were also sparse and thin (fig 2). Her teeth were small and widely spaced, numbering 25 in all. Cardiac murmuro was not heard. Development of the breasts, axillary

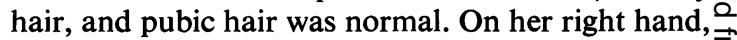
the second finger was missing, and the third fingero consisted of only the proximal phalanx. Campto- $\frac{3}{2}$ dactyly was noted in the first, fourth, and fifth fingers. On her left hand, the second finger was missing, the third finger had no distal phalanx, and the fourth finger had neither the distal phalanx nor.

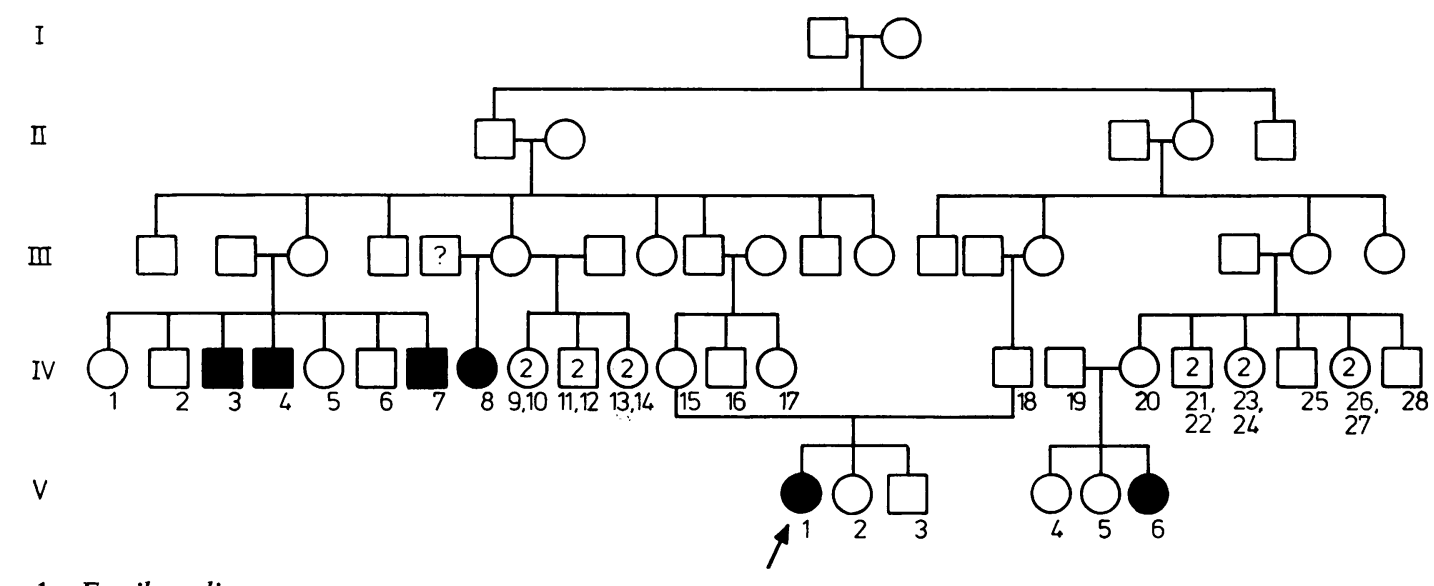

FIG 1 Family pedigree. 


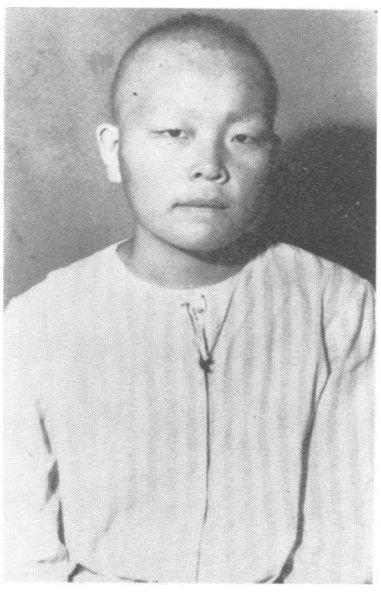

FIG 2 The proband.

the distal portion of the middle phalanx. Furthermore, $x$-ray showed syndactyly between the third had fourth fingers (fig 3). On her right foot, the distal phalanx of the second toe was divided into two,

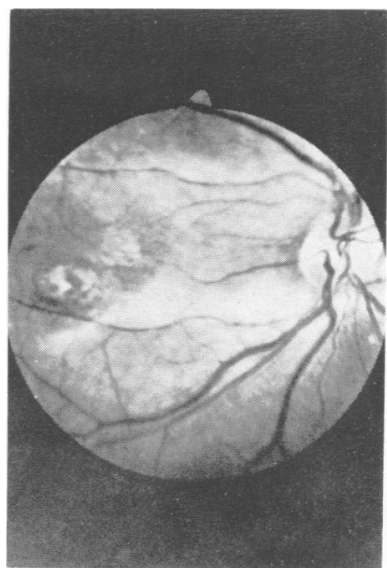

FIG 4 Ocular fundus of the proband. attributable diagnostically to the combination of polydactyly and syndactyly (fig 3 ). The toes on her left foot were normal.

In her ocular fundi (fig 4), retinochoroidal atrophy
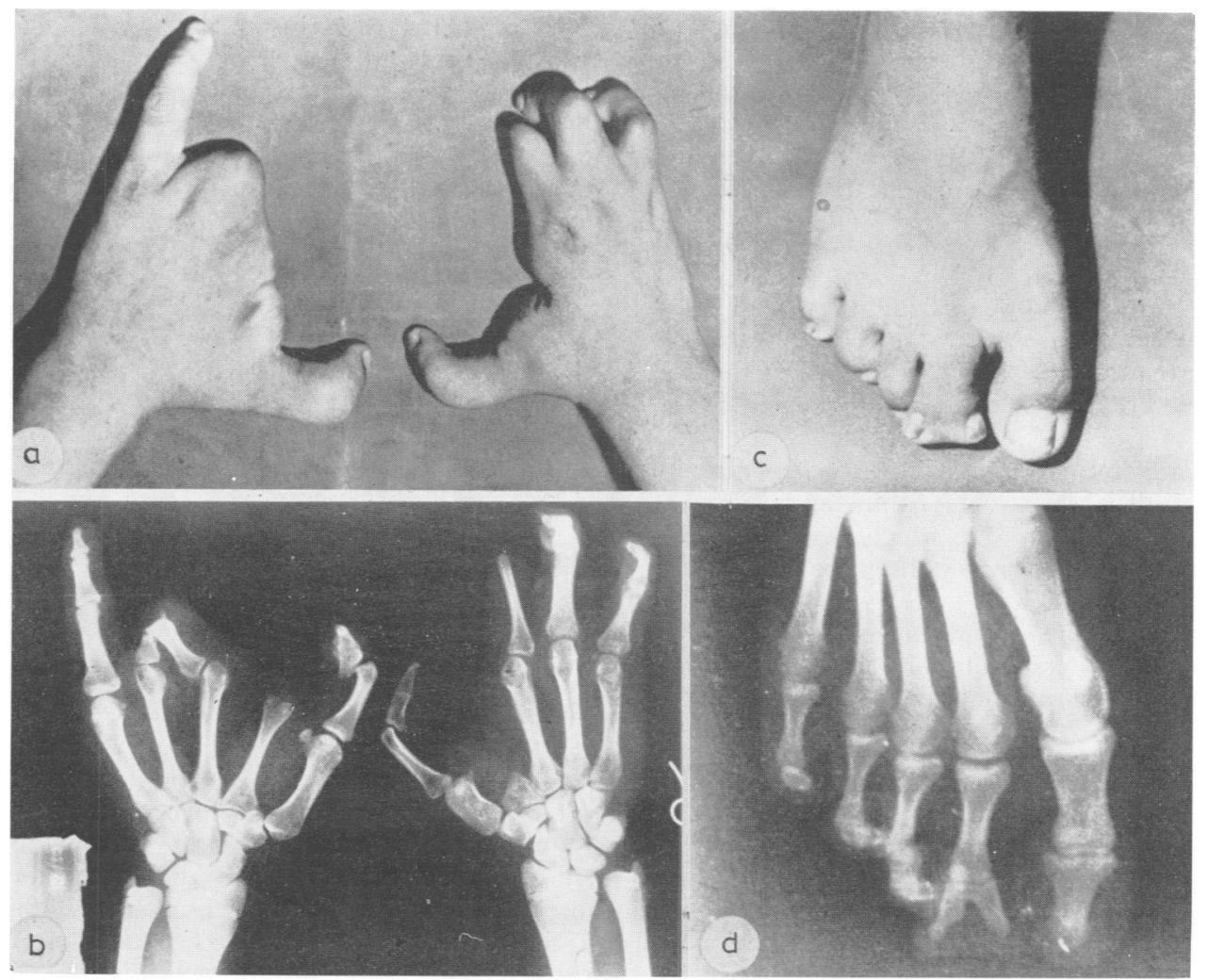

FIG 3 (a) Dorsal aspect of the hands, (b) $x$-ray of the hands, (c) the right foot, and (d) $x$-ray of the right foot of the proband. 


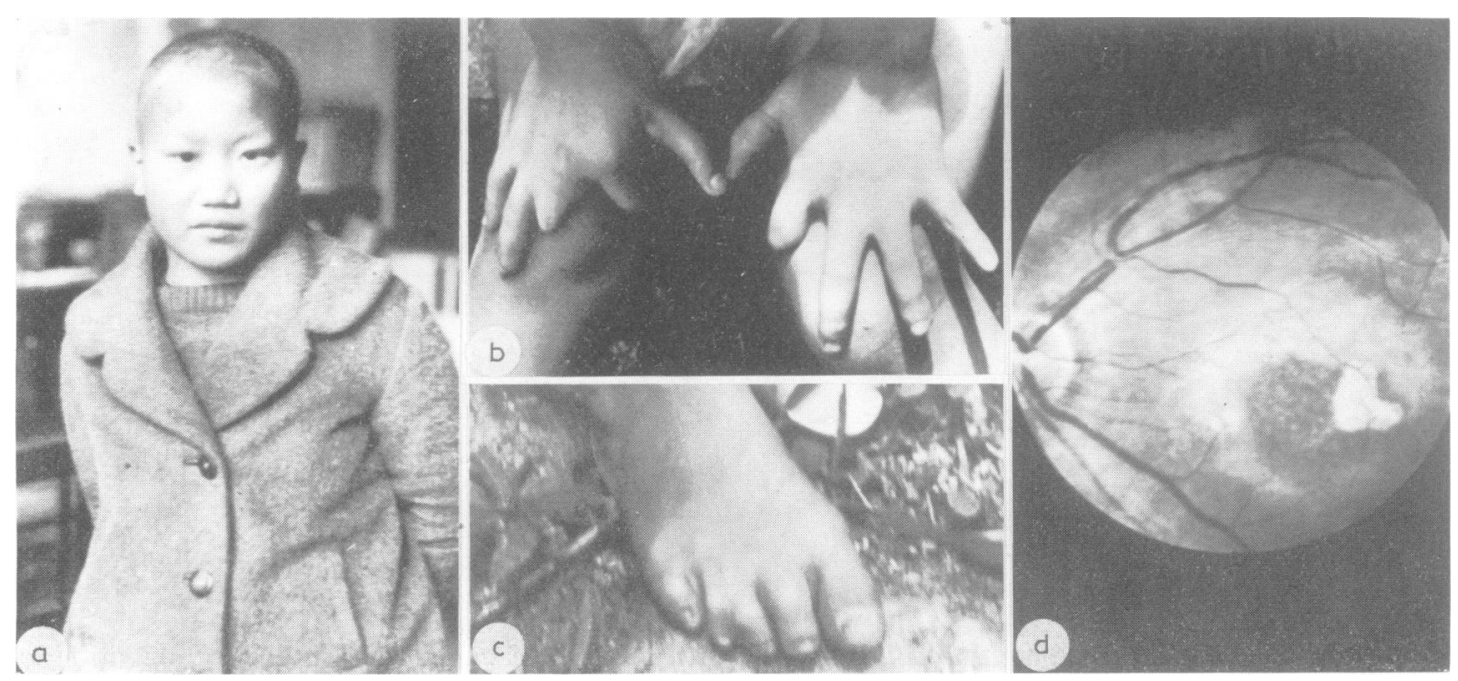

FIG 5 (a) Front view, (b) hands, (c) right foot, and (d) ocular fundus of case 2.

was observed in the macular region. Four small areas (1/4 disc size) of pigment mottling of yellowish-white colour were noted in the retinal deep layer around the fovea. The peripheral retina and vessels were normal. Ths test for colour vision revealed deuteranomalopia.

The sweat test was normal, IQ was 98 as determined by the WAIS method, and the lymphocyte karyotype was normal.

\section{CASE 2}

$\mathrm{V} \cdot 6$ (fig 1) was a girl aged 10 years 8 months when we examined her. Her parents were distantly related and had no malformations or visual disturbance. Her two sisters were healthy. She is a second cousin of the proband.

Her height and weight were within the normal range. Her hair was very sparse, thin, and short (fig 5). She had 19 teeth in total which were widely spaced. Breast development was normal for her age. No cardiac murmur was heard.

On her right hand, the second finger was missing from the proximal interphalangeal joint, and the third finger was missing from the portion corresponding to the body of the proximal phalanx. On her left hand, the second finger was missing from the proximal interphalangeal joint. Syndactyly between the third and fourth toes on her right foot was noted (fig 5).

In the fundi, retinochoroidal atrophy without pigment mottling was observed centring on the macula (fig 5). Sweating was normal.

CASE 3

IV.8 (fig 1) was a 39-year-old woman when we examined her. She was illegitimate. Though her father was not known, it was rumoured that he was one of the villagers. She was a first cousin once removed of the proband.

Her clinical features were similar to those of the proband. The distal phalanx of the second finger of both hands was missing, but her toes were normal.

In the fundi, extensive retinochoroidal atrophy centring on the macula was observed, within which $\stackrel{\square}{\circ}$ pigment mottling was scattered (fig 6). She refused $\underset{\vec{B}}{\overrightarrow{7}}$ further examination and photographs.

CASE 4

IV.3 (fig 1) was a 45-year-old man when we examined him. He is a second cousin of the proband and his parents were distantly related. Although they were dead, we were told that they had no ectrodactyly or 3 . visual disturbance. He had six sibs, of whom two brothers showed the same malformations as he. However, one of them (IV.7, fig 1) had died ino infancy.

His physique was normal. His hair was hardlyo visible and his eyebrows and eyelashes were very scanty (fig 7).

He had only the first and fifth fingers on each $\tilde{O}^{-}$ hand. On the first finger of the right hand, a process $N$ which looked like the second finger was notedo (fig 7). On his left foot, the second toe was missingo and syndactyly between the third and fourth toes was observed. The fifth toe was hypoplastic. On his? right foot, syndactyly between the first and second toes was detected and the third toe was missing. The fourth and fifth toes were hypoplastic (fig 7).

In both fundi, extensive retinochoroidal atrophy

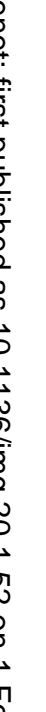




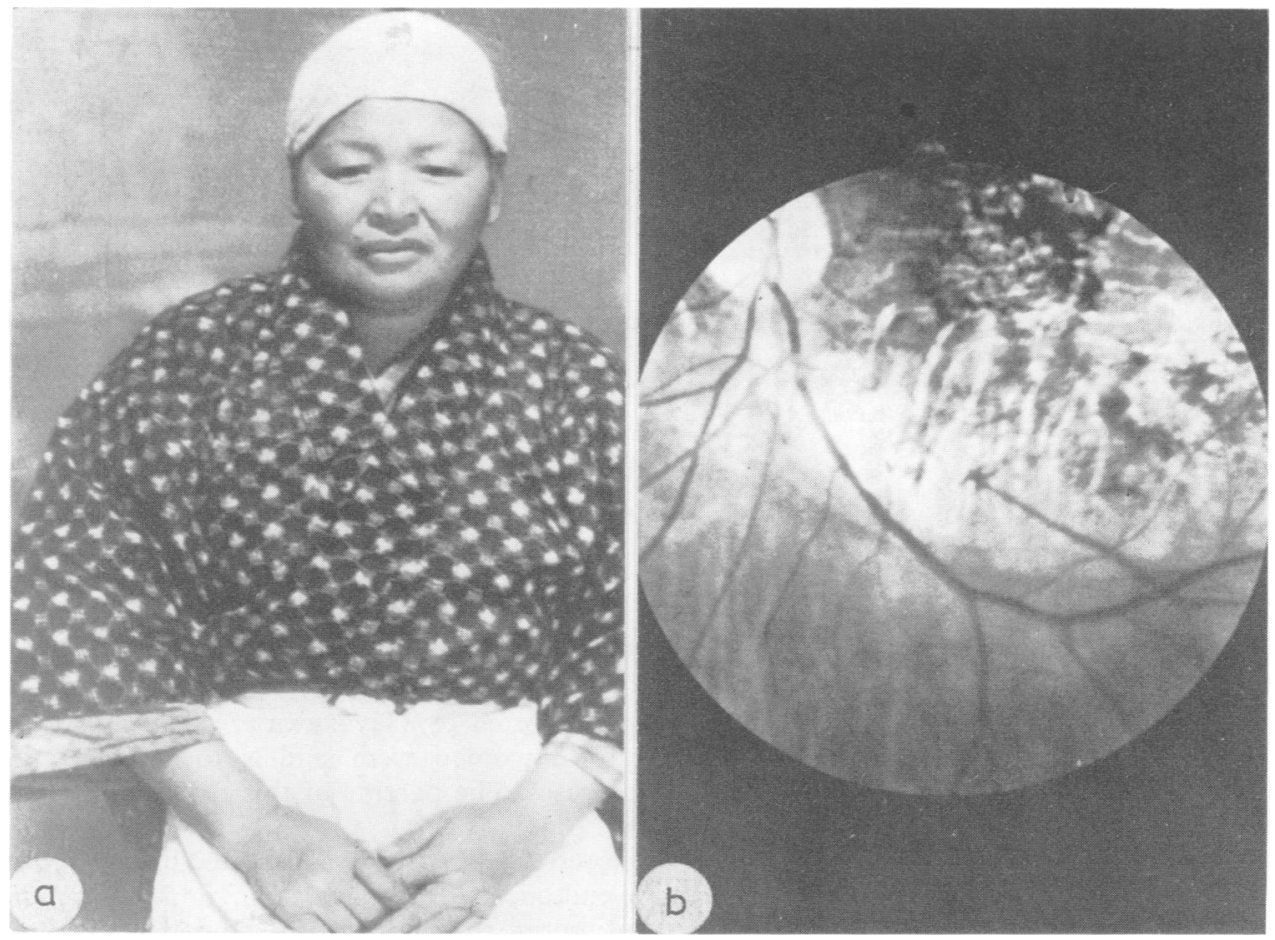

FIG 6 (a) Front view and (b) ocular fundus of case 3.

was noted in the posterior pole, within which pigment mottling was seen. The optic discs were atrophic. The retina in the periphery was normal (fig 7).
CASE 5

IV. 4 (fig 1) was a 42-year-old man when we examined him. He is a brother of case 4 . His clinical findings were similar to those of case 4 (fig 8 ).
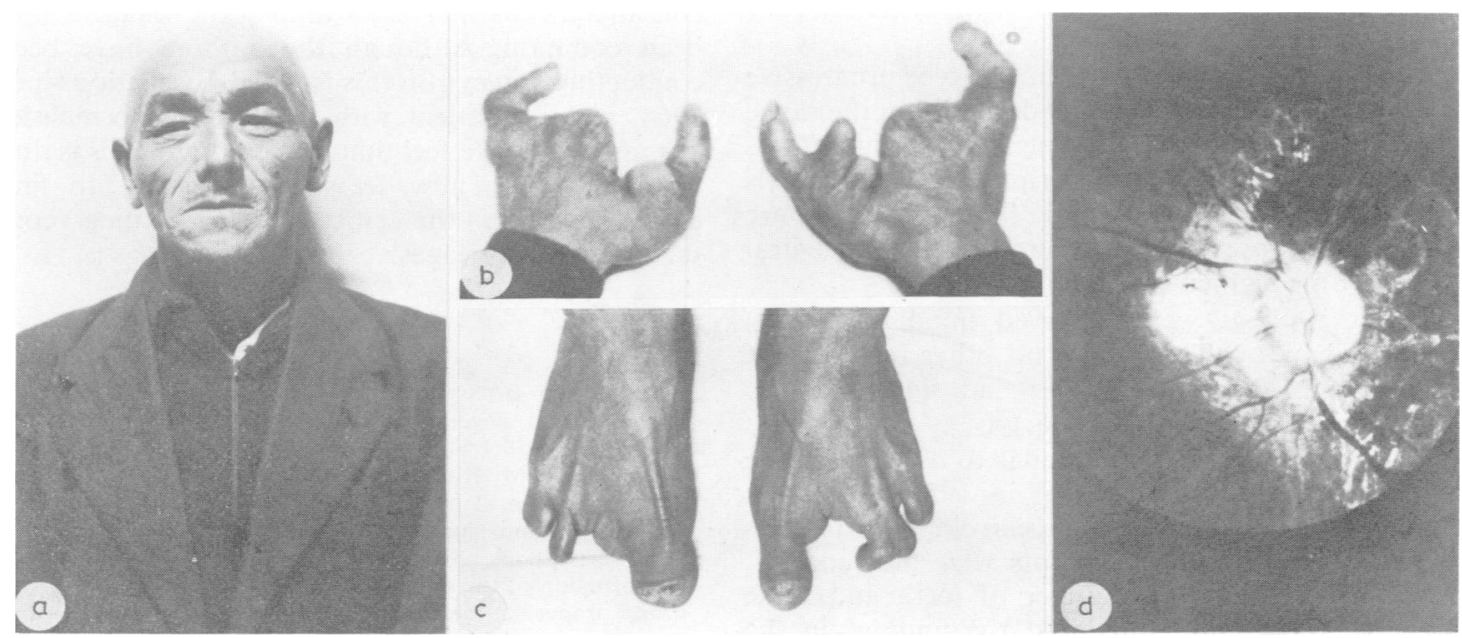

FIG 7 (a) Front view, (b) hands, (c) feet, and (d) ocular fundus of case 4. 


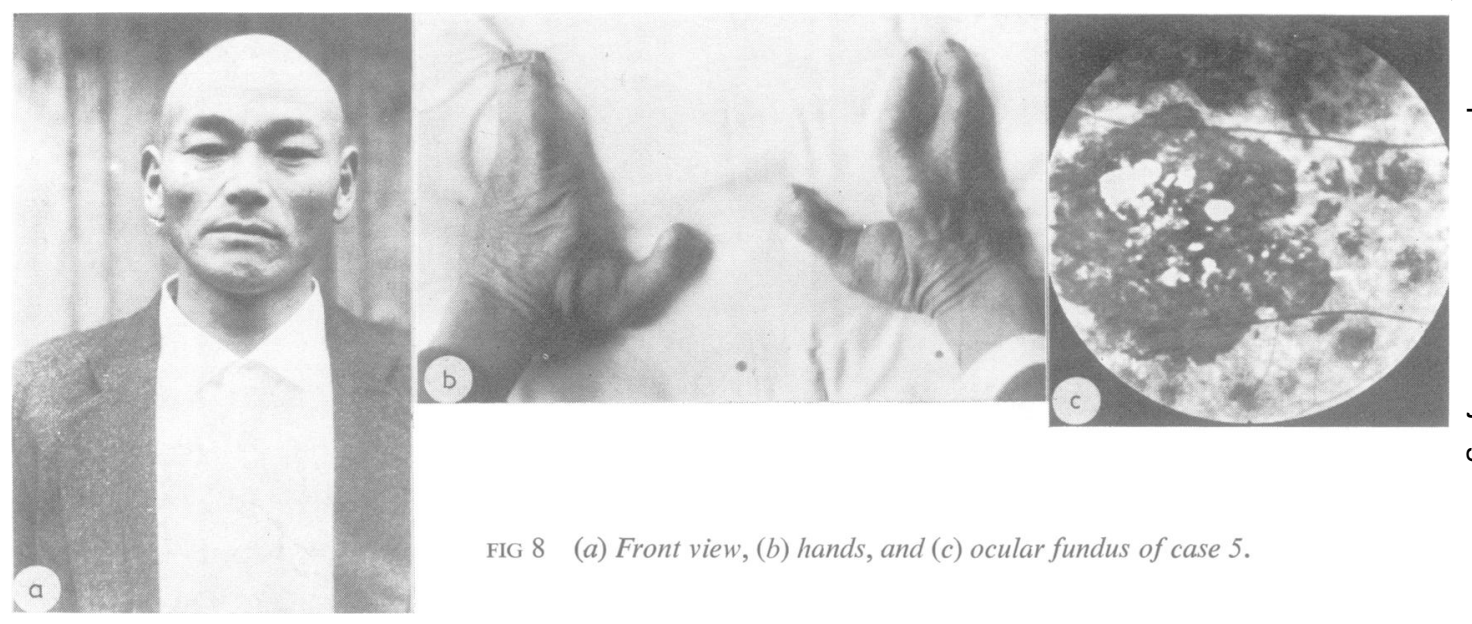

On his right hand, the second and fifth fingers were missing. On his left hand, the second and fifth fingers were missing and there was syndactyly between the third and fourth fingers (fig 8). The toes were normal.

The findings in the fundus were similar to those in case 4 (fig 8).

\section{Discussion}

The cases described in this paper show characteristic combinations of the following congenital abnormalities.

(1) The hair, eyebrows, and eyelashes were very sparse. Partial anodontia was observed and the teeth were small.

(2) Ectrodactyly associated with syndactyly or cleft hand or both was noted.

(3) Macular dystrophy presumed to be progressive was observed in the ocular fundus. Within the range of the macular dystrophy, pigment mottling was scattered. The retina in the periphery and the vessels in the fundus were normal. These findings are similar to those found in central and pericentral retinopathia pigmentosa.

These findings were observed in all five cases examined by us. There were no other members of this isolated community who had signs of ectodermal dysplasia or of ectrodactyly.

The first report of cases similar to ours was made by Albrectsen and Svendsen ${ }^{1}$ in 1956. The cases reported by them were a 4-year-old boy and his 6-year-old sister, whose parents were first cousins. They had the normal number of teeth and these researchers did not think that the findings in the fundus were progressive. Only these findings differ from those of the cases described in this paper. The findings reported by Hayakawa $e t a l^{2}$ in 1979 of a 30-year-old woman were similar to those of the present cases. The parents of this patient were first cousins.

If we add together the cases reported by Albrectsen and Svendsen, ${ }^{1}$ by Hayakawa et $a l,{ }^{2}$ and those in the present report, the number of male patients is three and the number of female patients is five. All their parents were consanguineous. Consequently, we attribute the aetiology to an autosomal recessive trait.

The isolated population in which the authors found $\frac{0}{3}$ the present cases started with 30 families some $300 ?$ years ago. These 30 families originated from three families living in a very remote village. Thereafter, consanguineous marriages among the villagers have been common. Although the authors have been $\frac{\sigma}{3}$ conducting surveys on this isolated population since 1964, no new patient with the same abnormalities $ᄋ$ has appeared. We feel that the reason for this is that the youth are now leaving the island to find 9 employment in the cities, thus avoiding con- $>$ sanguineous marriages.

\section{References}

1 Albrectsen B, Svendsen IB. Hypotrichosis, syndactyly, N and retinal degeneration in two siblings. Acta Derm Venereol 1956;1:96-101.

2 Hayakawa M, Kato K, Yamauchi Y. A case of central $\stackrel{Q}{C}$ and pericentral retinopathia pigmentosa with abnor- $\mathbb{D}$ malities of hair, hands and teeth. Ganka $1979 ; 21: 433-8$.

${ }^{3}$ Roberts JB. A child with double cleft of lip and palate, $\square$ protrusion of the intermaxillary portion of the upper jaw and imperfect development of the bones of the four $\frac{\vec{D}}{d}$

extremities. Ann Surg 1919;70:252-3.
4 Rudiger RA, Haase W, Passarge E. Association of $\frac{\rho}{\mathscr{\Phi}}$ 
ectrodactyly, ectodermal dysplasia, and cleft lip-palate. The EEC syndrome. Am J Dis Child 1970; 120:160-3.

5 Robinson GC, Miller JR, Bensimon JR. Familial ectodermal dysplasia with sensori-neural deafness and other anomalies. Pediatrics 1962;30:797-802.

6 Freire-Maia N. A newly recognized genetic syndrome of tetramelic deficiencies, ectodermal dysplasia, deformed ears, and other abnormalities. Am J Hum Genet 1970;22 : $370-7$.
7 Bowen P, Armstrong HB. Ectodermal dysplasia, mental retardation, cleft lip/palate and other anomalies in three sibs. Clin Genet 1976;9:35-42.

Requests for reprints to Dr S Ohdo, Department of Pediatrics, Miyazaki Medical College, 5200 Kihara, Kiyotake-cho, Miyazaki, Japan. 